\title{
High Spatial and Spectral Resolution Long-Range Sensing Using Brillouin Echoes
}

\author{
Stella M. Foaleng, Moshe Tur, Fellow, IEEE, Jean-Charles Beugnot, and Luc Thévenaz
}

\begin{abstract}
High spatial $(\sim \mathrm{cm})$ and spectral $(\sim \mathrm{MHz})$ resolution Brillouin sensing is realized with enhanced signal to noise ratio using a pre-activated acoustic field and an optical phase control over the interrogating pulse. Pre-activation of the acoustic field preserves the Brillouin natural linewidth and a differential gain technique extends the method to long ranges. Experimentally, fully resolved measurements of the Brillouin frequency shift of a $5 \mathrm{~cm}$ spot perturbation at the far end of a $5 \mathrm{~km}$ fiber have been performed with a frequency resolution of $3 \mathrm{MHz}(2 \sigma)$, using a $500 \mathrm{ps}$ $(5 \mathrm{~cm}) \pi$ phase shift pulse.
\end{abstract}

Index Terms-Brillouin echoes, distributed fiber sensor, fiber optics sensors, stimulated Brillouin scattering.

\section{INTRODUCTION}

$\mathbf{F}$ OR at least two decades, the interaction between acoustic and light waves, observed trough the optical process called Brillouin scattering, has attracted substantial attention in the fiber sensing community, since it turns out that Brillouin scattering is particularly efficient and attractive for the implementation of strain and temperature distributed sensing in optical fibers [1]-[3]. When it is spontaneous, a pump wave propagating in the fiber is scattered by thermally activated acoustic phonons in the fiber into a back propagating wave, which is spectrally shifted from the pump frequency by $\nu_{B}=2 n V_{a} / \lambda_{p}$ (where $n$ is the refractive index of the fiber, $V_{a}$ the acoustic velocity and $\lambda_{p}$ is the wavelength of the pump) over a very narrow band (a few tens of $\mathrm{MHz}$ ) as a result of the strict phase matching condition required by the interaction. Being intrinsically very sensitive to the temperature and strain, this frequency shift can be used to map the local temperature and strain along the fiber.

When Brillouin scattering is stimulated (SBS), counterpropagating pump and signal waves, spectrally separated by the Brillouin shift frequency $\nu_{B}$, interfere and produce through electrostriction an acoustic field, which in turn initiates a power

Manuscript received March 22, 2010; revised July 23, 2010, August 19, 2010; accepted August 29, 2010. Date of publication September 07, 2010; date of current version October 04, 2010. This work was supported in part by Omnisens and in part by the COST Action 299 "FiDES." The work of M. Tur was supported in part by the Israel Science foundation (ISF)

S. M. Foaleng, J.-C. Beugnot and L. Thévenaz are with the Institute of Electrical Engineering, École Polytechnique Fédérale de Lausanne, CH-1015, Lausanne, Switzerland (e-mail: stella.foalengmafang@epfl.ch; luc.thevenaz@epfl. ch).

M. Tur was with the Institute of Electrical Engineering, École Polytechnique Fédérale de Lausanne, CH-1015, Lausanne, Switzerland. He is now with the Faculty of Engineering, Tel-Aviv University, 69978 Israel (e-mail: tur@eng. tau.ac.il).

Color versions of one or more of the figures in this paper are available online at http://ieeexplore.ieee.org.

Digital Object Identifier 10.1109/JLT.2010.2073443 transfer between the two optical waves. Here, changes in strain or temperature can be determined as well by locally measuring the central frequency of the Brillouin interaction spectrum at any position along the fiber. Position information is resolved from a classical time-of-flight conversion from the temporal traces of the signal wave backpropagating in the fiber.

Instruments based on spontaneous Brillouin for Time Domain Reflectometry (BOTDR) [4] require only one access point to the test fiber to launch the pump wave, while those using SBS for Brillouin Analysis (BOTDA) must access both ends of the test fiber to launch the pump pulse and CW signal, [1]-[3]. Both types of instruments offer good accuracy for temperature and strain measurements, albeit at spatial resolutions not better than $\sim 1 \mathrm{~m}$ [5]. The reason comes from the fact that the observed Brillouin spectrum broadens as the pulsewidth decreases, since the spectral distribution of the effective gain is given by the convolution between the pulse spectrum, broader for shorter pulses, and the natural Brillouin gain spectrum $(\sim 30 \mathrm{MHz}$ in silica fibers at $\lambda_{p}=1550 \mathrm{~nm}$, determined by the phonon lifetime $\tau_{A} \cong 6$ ns) [6]. This broadening leads to a reduced peak gain and uncertainties in the determination of the Brillouin shift $\nu_{B}$, and consequently to a reduced accuracy in the measurement of temperature and strain at submetric spatial resolutions. A natural limit is given by the situation when pump pulse and Brillouin gain spectra show comparable bandwidth, corresponding to a pump pulse showing a duration equal to a double phonon lifetime $2 \times \tau_{A} \cong 12 \mathrm{~ns}$, or $\sim 1$ meter spatial resolution.

However, modern advanced applications require a substantial step towards better spatial resolution, while preserving temperature/strain precision over a long range. For this purpose frequency-domain and correlation-based techniques have been developed to circumvent the phonon lifetime limitations and exhibit enhanced spatial resolutions down to $40 \mathrm{~cm}$ and $1 \mathrm{~cm}$, respectively [7], [8]. Nevertheless, both these techniques introduce their own complexities, including a tradeoff between spatial resolution and range.

Recently, a radically new approach has been presented for time domain measurements, relying on the pre-activation of the acoustic field within the Brillouin frequency resonance. First observed by Bao et al. [9], high spatial resolution could be obtained this way, while keeping the Brillouin gain profile at its natural narrow width. In this approach, the pre-activated acoustic field is electrostrictively generated through the interference between a CW pump wave (or a long pulse of duration $\gg \tau_{A}$ ), and a counter-propagating $\mathrm{CW}$ signal wave, properly frequency shifted from the pump wave. Once the acoustic wave has fully developed, a very fast change (shorter than $\tau_{A}$ ) in the pump signal can imprint a corresponding equally fast 
time-stamp on the amplified signal wave, without significantly affecting the acoustic field [10]. Being excited by $\mathrm{CW}$ (or quasi-CW) waves, the natural Brillouin linewidth is preserved and the observed time-stamp will have narrow frequency characteristics, resulting in high sensitivity to temperature and stain, while simultaneously providing high spatial resolution determined by the fast features of the time stamp.

Several options exist as to the nature of the fast change imposed on either the pump or signal waves. A spatial resolution of $15 \mathrm{~cm}$ was demonstrated using a bright pulse on top of a continuous wave [9], [11], while a dark pulse technique, i.e., shutting down one of the $\mathrm{CW}$ waves for a very brief time, could resolve 2 $\mathrm{cm}$ [12]. A variant of the bright pulse technique, PPP-BOTDA [13], employs a Pulse Pre-Pump of lower intensity to activate the acoustic field, before a stronger interrogating pulse arrives. By analyzing the physical principles behind these processes, a more recent configuration has been proposed, which optimizes the response by modulating the pump using a $\pi$ phase pulse, a configuration we call a Brillouin Echo Distributed Sensing (BEDS) [14], [15]. This denomination comes from the formal analogy in the equations with the spin echoes as observed in nuclear magnetic resonance and more straightforwardly highlights the physical fact that the time stamp on the pump experiences a pure reflection on the acoustic wave and is thus echoed on the signal.

The implementation of BEDS is realized by propagating continuous pump and signal waves through the sensing fiber at the proper frequencies for the Brillouin interaction and by abruptly and briefly shifting the phase of the CW pump wave by $\pi$ during a time much shorter than the phonon lifetime $\tau_{A}$. During this very short time the acoustic wave doesn't have time to noticeably change its phase and amplitude, so that the pump will be reflected completely out of phase during the brief $\pi$ phase shift, when compared to the normal steady-state gain situation. A destructive interference on the signal results from this pump reflection, observed as a small apparent loss on the signal waveform. This brief reflection on the pre-existing acoustic wave is called the first echo and its magnitude is proportional to the amplitude of the steady acoustic wave generated by the continuous counter-propagating optical waves. When the phase of the pump returns to its initial state, the normal steady state gain situation is restored. Nevertheless, the acoustic wave slightly changed its amplitude and phase during the pump phase pulse and then returns slowly to its initial state during a typical time on the order of a few $\tau_{A}$. This creates a small deficit in gain during this period, which manifests itself as a trailing and decaying loss over the signal waveform. This effect is observed as a long lasting second echo and is clearly detrimental for the measurement, since it gives a trailing signal that may interfere at later positions with the correct information given by the first echo. While the relative importance of the second echo, when compared to the first main echo, decreases for shorter pulses, it is highly desirable to completely eliminate its effects.

In this paper, we further investigate the Brillouin back-scattered signals, following $\pi$ phase shifted pump pulses, and show an optimized implementation that gives real metrological measurements, while detailing the reasons of the physical response of the system. Experimentally, we demonstrate a spatial res- olution down to $5 \mathrm{~cm}$ (with only 256 averages), with a Brillouin linewidth identical to the natural one, thereby simultaneously achieving high sensitivity for temperature/strain measurements and high spatial resolution. Using a double pulse technique, previously shown in our earlier paper, [14], and later judiciously implemented in a pure intensity-based scheme [16], second echoes can be totally screened and the $5 \mathrm{~cm}$ spatial resolution capabilities of BEDS are extended to $5 \mathrm{~km}$ without pump depletion penalty.

\section{THEORETICAL DESCRIPTION}

The process of stimulated Brillouin scattering involves an electrostrictively-induced acoustic wave, generated through the interference between the pump and signal waves. Mathematically, the assumed co-polarized but counter-propagating pump and signal waves, $A_{p}(z, t) \exp \left[i\left(k_{p} z-\omega_{p} t\right)\right]$ and $A_{s}(z, t) \exp \left[i\left(-k_{s} z-\omega_{s} t\right)\right]$, will generate an acoustic field, $Q(z, t) \exp [i(q z-\Omega t)], q=k_{p}+k_{s}, \Omega=\omega_{p}-\omega_{s}$, which will then couple the two optical fields according to [17]:

$$
\begin{aligned}
\frac{\partial A_{p}(z, t)}{\partial z}+\frac{1}{V_{g}} \frac{\partial A_{p}(z, t)}{\partial t} & =i \frac{1}{2} g_{2} A_{s}(z, t) Q(z, t) \\
\frac{\partial A_{s}(z, t)}{\partial z}-\frac{1}{V_{g}} \frac{\partial A_{s}(z, t)}{\partial t} & =-i \frac{1}{2} g_{2} A_{p}(z, t) Q^{*}(z, t) \\
\frac{\partial Q(z, t)}{\partial t}+\Gamma_{A} Q(z, t) & =i g_{1} A_{p}(z, t) A_{s}^{*}(z, t) \\
\Gamma_{A} & =i \frac{\Omega_{B}^{2}-\Omega^{2}-i \Omega \Gamma_{B}}{2 \Omega}
\end{aligned}
$$

Notations and approximations: $\omega_{p}, \omega_{s}$ and $\Omega=\omega_{p}-\omega_{s}$, are the center angular frequencies of the pump, signal and acoustic waves, respectively, while $k_{p}, k_{s}$ and $q=k_{p}+k_{s}$ are their wavenumbers. $g_{1}\left(=\gamma_{e} q^{2} /(8 \pi \Omega)\right)$ and $g_{2}$, represent, respectively, the electrostrictive and elasto-optic coupling constants [17]. $A_{p}(z, t), A_{s}(z, t)$ and $Q(z, t)$ are slowly varying complex envelopes. $\Omega_{B}=2 \pi \nu_{B}$ is the Brillouin frequency shift for resonant interaction between the three waves, and $\Gamma_{B}=1 / \tau_{A}=$ $2 \pi \Delta \nu_{B}$ is the acoustic damping constant, intimately related to the phonon life time $\tau_{A}$ and the FWHM width of the Brillouin gain spectrum $\Delta \nu_{B}$. It must be pointed out here that the Brillouin gain spectral distribution is determined by the response to the acoustic wave amplitude $Q$, as evidently apparent in (1), while the phonon lifetime $\tau_{A}$ is defined by the acoustic energy loss [17]. As a result of the square law dependence between amplitude and energy, the rate of change on the acoustic amplitude is scaled by twice the phonon lifetime. This also explains the factor 2 in the expression relating the phonon lifetime $\tau_{A}$ to the FWHM width $\Delta \nu_{B}$, which is frequently omitted in the literature. By excluding derivatives with respect to $z$ in (1c) [17], we have assumed that an induced acoustic disturbance at $\left(z_{0}, t_{0}\right)$ will remain spatially local and will not propagate along the fiber. This common approximation is quite justified in our case, where due to the relatively minuscule value of the acoustic velocity with respect to that of light, the generated acoustic envelope at $z_{0}$ barely moves $(<100 \mu \mathrm{m})$, while a pump pulse, as long as ten nanoseconds, completes its voyage over $z_{0}$, thereby ceasing 
its interaction with the practically spatially static acoustic disturbance at that point.

Let assume a constant signal wave, $A_{s}(z, t) \equiv A_{s}^{0}$, null initial acoustic conditions, $Q(z, t=0) \equiv 0$, and a propagating pump pulse of temporal width, $T$. At some arbitrary point along the fiber, $z_{0}$, the time evolution of the complex envelope at $\Omega=$ $\omega_{p}-\omega_{s}, Q\left(\Omega, z_{0}, t\right)$ is given by the direct solution of $(1 \mathrm{c})$ in (2) shown at the bottom of the page.

The dynamic characteristics of the solutions (1) heavily depend on $\operatorname{Re}\left(\Gamma_{\mathrm{A}}\right)=\Gamma_{\mathrm{B}} / 2$. We'll, therefore, examine two extreme cases.

a) For a pump pulse much longer than $1 / \operatorname{Re}\left(\Gamma_{A}\right)=$ $2 / \Gamma_{B}=2 \tau_{A}, Q\left(\Omega, z_{0}, t\right)$ of (2a) reaches its maximum magnitude long before the pulse is over, attaining a frequency-dependent value of

$$
\begin{aligned}
&\left|Q\left(\Omega, z_{0}, T\right)\right|=\frac{\left|g_{1} A_{p}^{\text {pulse peak }} A_{s}^{0 *}\right|}{\left|\Gamma_{A}\right|} \\
& \approx \frac{\left|g_{1} A_{p}^{\text {pulse peak }} A_{s}^{0 *}\right|}{\sqrt{\left(\Omega-\Omega_{B}\right)^{2}+\left(\Gamma_{B} / 2\right)^{2}}} \\
& \text { for } T \gg \frac{2}{\Gamma_{B}}
\end{aligned}
$$

In classical Brillouin distributed sensing $\Omega$ is scanned through a range of optical frequencies to precisely determine $\Omega_{B}$, range information is obtained from the pulse time of flight data, and the spatial resolution is determined by the pulse width. According to (3), long pulses, while having very poor spatial resolution, will give rise to acoustic field amplitudes sharply peaked at $\Omega=\Omega_{B}$, allowing the sensor to resolve local temperature (and/or strain) induced changes in $\Omega_{B}$ with a sensitivity limited only by the natural Brillouin linewidth, $\Gamma_{B}$ and that of the processing electronics.

b) For a pump pulse shorter than $1 / \operatorname{Re}\left(\Gamma_{A}\right)=2 / \Gamma_{B}=2 \tau_{A}$, (2a) can be approximated in the vicinity of $\Omega_{B}$ (i.e., when $\left|\Omega_{B}-\Omega\right| T<1$ ) by

$$
\begin{aligned}
\left|Q\left(\Omega, z_{0}, T\right)\right| & \approx\left|\frac{i g_{1} A_{p}^{\text {pulse peak }} A_{s}^{0 *}}{\Gamma_{A}} \cdot\left(\Gamma_{A} T\right)\right| \\
& =\left|g_{1} A_{p}^{\text {pulse peak }} A_{s}^{0 *} T\right| \text { for } T \ll 2 / \Gamma_{B} .
\end{aligned}
$$

Equation (4) clearly states that the magnitude of the acoustic field, $\left|Q\left(\Omega, z_{0}, T\right)\right|$, does not depend on $\Omega$ in the vicinity of $\Omega_{B}$. Here, high spatial resolution of the sensor is achieved at the expense of losing all spectral sensitivity!

This analysis has led manufacturers of Brillouin time domain analyzers to compromise on pump pulse widths not shorter than $5 \mathrm{~ns} \sim 1 / \Gamma_{B}$, limiting the spatial resolution of the instrument to $\sim 0.5 \mathrm{~m}$

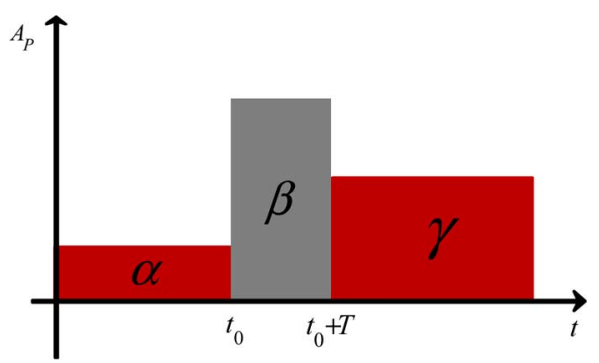

Fig. 1. Pump coding waveform, where $\alpha, \beta$, and $\gamma$ can be either real or complex. The duration of the $\beta$ part of the pump pulse, $T$, will determine the spatial resolution.

As described in the introduction, recent developments have concentrated on separating the generation of the acoustic field from its probing by superimposing a pulse on the CW pump. This scenario can be mathematically investigated by solving the three coupled (1). Elsewhere, [18], we have obtained an analytical solution for a $\mathrm{CW}$ input signal, and a pump wave comprising three concatenated square pulses having arbitrary (including complex) amplitudes and durations, see Fig. 1, representing the general situation of a time-domain pulse coding, so that a solution covering all configurations reported in the literature can be established. We also assume an unattenuated, undepleted pump wave and small Brillouin gain, so that the signal wave amplitude, $A_{s}(z, t)$, can be approximated using a perturbation approach by $A_{s}(z, t)=A_{s}^{0}+a_{s}(z, t)$ with $\left|a_{s}(z, t)\right| \ll$ $\left|A_{s}^{0}\right|$.

Under these assumptions (1a) does not need to be solved, and the other equations reduce to

$$
\begin{aligned}
\frac{\partial a_{s}(z, t)}{\partial z}-\frac{1}{V_{g}} \frac{\partial a_{s}(z, t)}{\partial t} & =-i \frac{1}{2} g_{2} A_{p}(z, t) Q^{*}(z, t) \\
\frac{\partial Q(z, t)}{\partial t}+\Gamma_{A} Q(z, t) & =i g_{1} A_{p}(z, t) A_{s}^{0 *} .
\end{aligned}
$$

With an eye on the experimental setup of Section IV below, we'll analyze here a particular situation, where a $5 \mathrm{~cm}$ fiber section with $\Omega_{B}(5 \mathrm{~cm}) / 2 \pi=10.86 \mathrm{GHz}$ is spliced between two longer sections, $20 \mathrm{~m}$ each, of a different fiber, having $\Omega_{B}(20 \mathrm{~m}) / 2 \pi=10.73 \mathrm{GHz}$. Both fibers share a Brillouin bandwidth of $27 \mathrm{MHz}$. The power of the signal, as it emerges from the fiber is given by:

$$
\begin{aligned}
P_{s}(z=0, \Omega, t) & \propto\left|A_{s}\right|^{2}=\left|A_{s}^{0}+a_{s}\right|^{2} \\
& \approx\left|A_{s}^{0}\right|^{2}+2 \operatorname{Re}\left[A_{s}^{0 *} a_{s}(z=0, \Omega, t)\right] \\
& \equiv\left|A_{s}^{0}\right|^{2}+P_{\text {Brillouin }}
\end{aligned}
$$

Fig. 2 presents the results for the pulse of Fig. 1 with $t_{0} \gg \tau_{A}$ (to allow full pre-activation of the acoustic field), $T=500 \mathrm{ps}$ and $\Omega=\Omega_{B}(5 \mathrm{~cm}) / 2 \pi=10.86 \mathrm{GHz}$. Results for the Brillouin

$$
Q\left(\Omega, z_{0}, T\right)=\left\{\begin{array}{lll}
\frac{i g_{1} A_{p}^{\text {pulse peak }} A_{s}^{0 *}}{\Gamma_{A}}\left[1-\exp \left(-\Gamma_{A} t\right)\right] & t \leq T \quad(\mathrm{a}) \\
\frac{i g_{1} A_{p}^{\text {pulse peak }} A_{s}^{0 *}}{\Gamma_{A}} \exp \left(-\Gamma_{A} t\right)\left[\exp \left(+\Gamma_{A} T\right)-1\right] & t>T \quad(\mathrm{~b})
\end{array}\right.
$$




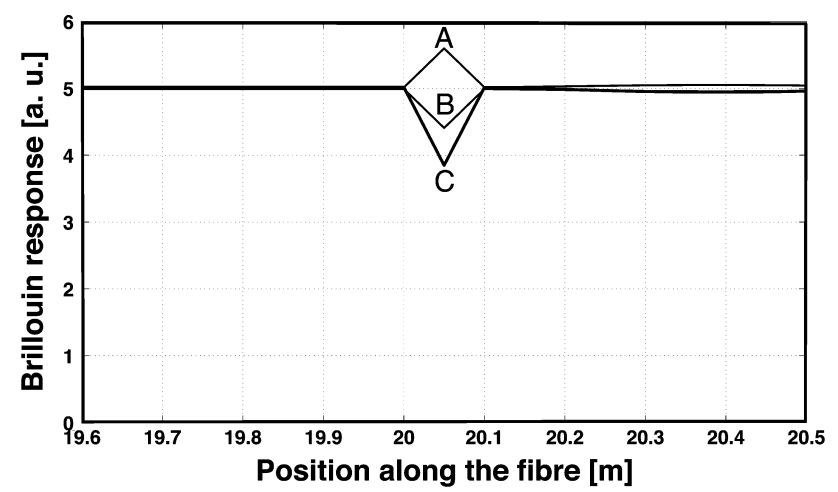

Fig. 2. Brillouin signal from a $5 \mathrm{~cm}$ section, spliced in the middle of a $40 \mathrm{~m}$ fiber, whose Brillouin frequency is detuned by $140 \mathrm{MHz}$ from that of the $5 \mathrm{~cm}$ section. $\Omega$ is tuned to the $5 \mathrm{~cm}$ section Brillouin frequency. The $\beta$ pump pulse is 500 ps long. A: Bright pulse; B: Dark pulse; $\mathrm{C}: \pi$ phase shift pulse.

signal, $P_{\text {Brillouin }}$, are shown for a bright pulse $(\alpha=1, \beta=$ $2, \gamma=1$, optimum setting), a dark pulse $(\alpha=1, \beta=0, \gamma=1)$ and a $\pi$ phase shift pulse $(\alpha=1, \beta=-1, \gamma=1)$.

Originally a function of time, the curves of Fig. 2 monitor the interaction of the narrow $\beta$ pump pulse with the counterpropagating signal wave. When the propagating narrow $\beta$ pulse resides in either of the $20 \mathrm{~m}$ sections (i.e., at distances outside the $5 \mathrm{~cm}$ section), it marginally affects the Brillouin signal, which is mainly due to the off-resonance $\left(\nu_{B}(5 \mathrm{~cm})-\nu_{B}(20 \mathrm{~m})=\right.$ $140 \mathrm{MHz}$ ) action of the $\alpha$ and $\gamma$ pulses in the two long $20 \mathrm{~m}$ sections, with a relatively small portion being contributed by the on-resonance but very short $5 \mathrm{~cm}$ section. Thus, as long as the $\beta$ pulse is away from the $5 \mathrm{~cm}$ section, the Brillouin signal can be expressed by (for clarity we have assumed that $A_{S}^{0 *}=1$ ):

$P_{\text {Brillouin }}(\beta$ pulse outside the $5 \mathrm{~cm}$ section)

$$
=2 \operatorname{Re}\left(a_{s, 2 \times 20 \mathrm{~m}}(\alpha, \gamma)+a_{s, 5 \mathrm{~cm}}(\alpha, \gamma)\right)
$$

$a_{s, 2 \times 20 \mathrm{~m}}(\alpha, \gamma)$ and $a_{s, 5} \mathrm{~cm}(\alpha, \gamma)$ are the Brillouin contributions of the $20 \mathrm{~m}$ and $5 \mathrm{~cm}$, respectively, and we ignored the contribution of the short detuned $\beta$ pulse to the Brillouin signal from the long sections. When the $\beta$ pulse resides in the $5 \mathrm{~cm} \mathrm{sec}-$ tion, the section contribution varies with the coding scheme, resulting in (note: $a_{s, 5} \mathrm{~cm}(\beta=1)=a_{s, 5} \mathrm{~cm}(\alpha=1$ or $\gamma=1)=$ $-a_{s, 5 \mathrm{~cm}}(\beta=-1)$ and $\left.a_{s, 5 \mathrm{~cm}}(\beta=2)=2 a_{s, 5} \mathrm{~cm}(\beta=1)\right)$ (8), shown at the bottom of the page. Thus, at the resonance frequency of the $5 \mathrm{~cm}$ section, Fig. 2, the Brillouin signal associated with this section (8) differs from the corresponding signal from the surrounding sections (7) by (9), shown at the bottom of the page.

Equation (9), as well as Fig. 2, clearly demonstrates that the $\pi$ phase shift pulse provides twice the contrast of the other methods. Thus, while all three coding schemes resolve the 5 $\mathrm{cm}$ section, the $\pi$ phase shift technique results in the highest signal-to-noise ratio. As $|\beta|$ increases, the performance of the bright pulse method will asymptotically approach that of the $\pi$ phase shift one.

The triangular shape of the calculated response from the $5 \mathrm{~cm}$ section is due to the fact that it takes time for the pulse to fully enter the section, and then to fully clear it. The initial buildup of the Brillouin pulse is not faster than the minimum between the pulse duration and the section length. In our example, they are identical: $5 \mathrm{~cm}$ are translated to $500 \mathrm{ps}$ and vice versa. Upon leaving the section the transition time is at least the pulse duration. Fig. 3 shows the Brillouin signal for the $\pi$ phase shift pump as a function of both the distance $\left(=V_{g} t / 2\right)$ and frequency detuning $\Omega\left(=\omega_{p}-\omega_{s}\right)$ for the same conditions as above. Similar to the other coding methods, the $5 \mathrm{~cm}$ section is well resolved not only in the time but also in frequency in spite of the very narrow probing pulse $\left(0.5 \mathrm{~ns}=T \ll \tau_{A}=6 \mathrm{~ns}\right)$. But the $\beta$ pulse, while probing the pre-excited acoustic field, also slightly modifies it, resulting in an observed trailing edge, designated as the second echo. This effect is clearly observed when the section under test is longer than the probing pulse. Consider Fig. 4, where the $5 \mathrm{~cm}$ section has been replaced by a $5 \mathrm{~m}$ one. During the entrance of the $\pi$ phase shift pulse (of duration $T$ ) into the 5 $\mathrm{m}$ section the signal level sharply drops (within $T$ ) to a level dictated by the Brillouin contributions of the $\beta$ pulse at the first 5 $\mathrm{cm}$ of the section and of the $\alpha$ pulse at the rest of the $5 \mathrm{~m}$ section (as well as the contributions from the $\alpha$ and $\gamma$ pulses at the $20 \mathrm{~m}$ sections). As the $\beta$ pulse continues its travel through the section, any length of fiber cleared by the $\beta$ pulse becomes populated by the $\gamma(=\alpha)$ pulse, and therefore, the signal level should not have changed. Instead, it keeps decreasing until it saturates after

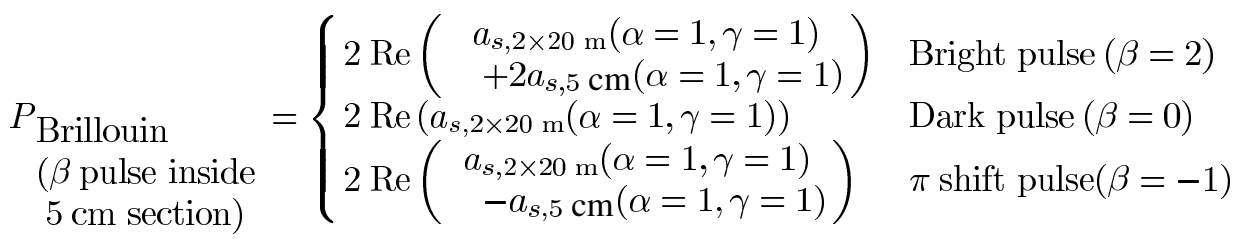

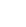

(n)

(1)

$$
\begin{aligned}
& P_{\text {Brillouin }}-P_{\text {Brillouin }} \\
& \begin{array}{ll}
(\beta \text { pulse inside } & (\beta \text { pulse outside } \\
5 \mathrm{~cm} \text { section }) & 5 \mathrm{~cm} \text { section })
\end{array}
\end{aligned}= \begin{cases}2 \operatorname{Re}\left(a_{s, 5 \mathrm{~cm}}(\alpha=1, \gamma=1)\right) & \text { Bright pulse }(\beta=2) \\
-2 \operatorname{Re}\left(a_{s, 5 \mathrm{~cm}}(\alpha=1, \gamma=1)\right) & \text { Dark pulse }(\beta=0) \\
-4 \operatorname{Re}\left(a_{s, 5} \mathrm{~cm}(\alpha=1, \gamma=1)\right) & \pi \text { shift pulse }(\beta=-1)\end{cases}
$$




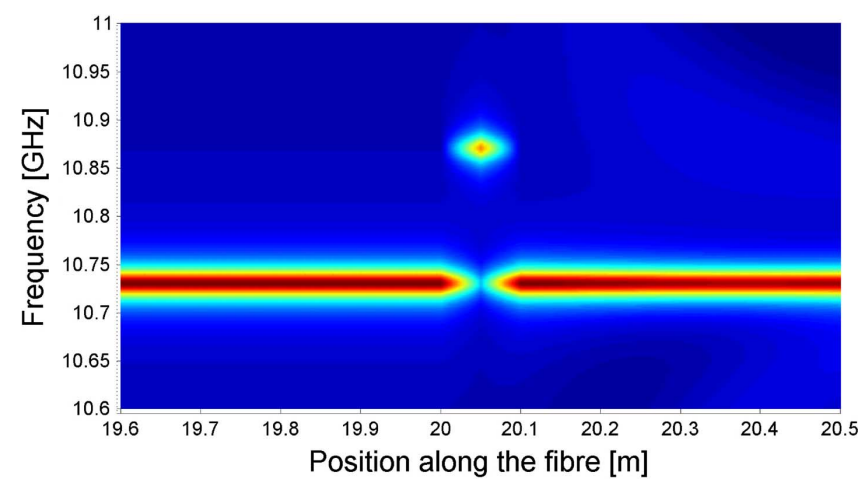

Fig. 3. A 2-D Distance-Frequency map of Brillouin gain from a $5 \mathrm{~cm}$ section, spliced in the middle of a $40 \mathrm{~m}$ fiber, whose Brillouin frequency is detuned by $140 \mathrm{MHz}$ from that of the $5 \mathrm{~cm}$ section. The $\Omega$ phase shift pulse is $500 \mathrm{ps}$ long. As in the experiment below, for each $\Omega$ the mean over time was removed and the redder the color the more negative are the values of the Brillouin signal (with respect to the signal initial power $\left|A_{S}^{0}\right|^{2}$.

a distance equivalent to a time lapse of a few tens of nanoseconds. This behavior is a direct consequence of the effect of the $\beta$ pulse on the pre-excited acoustic field: the $\gamma(=\alpha)$ pulse, which follows the moving $\beta$ pulse, meets a modified acoustic field, which is no longer fully matched to the counter-propagating pump and signal waves, and therefore, contributes less to the Brillouin signal. Saturation is achieved only after a few acoustic lifetimes when the $\beta$ pulse has propagated deep enough into the $5 \mathrm{~m}$ section so that the acoustic field at the beginning of the section has resumed its pre- $\beta$ pulse value. Once the $\beta$ pulse left the $5 \mathrm{~m}$ section, there is again a fast rise of the signal (of duration $T$ ) and then the above described process also manifests itself as a slow ascent of the signal to its value characteristic of the long sections: again, for a while, the $5 \mathrm{~m}$ section does not contribute its original share to the Brillouin signal. These manifestations of the second echo, and in particular the last one, may conceal true variations of $\Omega_{B}$, or may be erroneously identified as such. It must be mentioned that the demonstration shows fiber segments with spectrally well separated Brillouin gains. This choice was made to distinctly show the effect of the second echo on the response, but the second echo remains equally detrimental when the gains spectrally overlap. An improved pulse version of the BEDS method, with no secondary echoes will be described in Section VI.

\section{EXPERIMENTAL IMPLEMENTATION}

Our BEDS experimental configuration, shown in Fig. 5, is based on a simple modification of the high performance pump and probe setup described in [18]. A $1552 \mathrm{~nm} 11 \mathrm{~mW}$ compact external cavity laser diode (Redfern Integrated Optics PLANEX ${ }^{\mathrm{TM}}$ RIO0095) is used as the light source, having an enhanced coherence (linewidth $\Delta \nu=23 \mathrm{kHz}$ ). Actually a coherence time $\tau_{\mathrm{C}}=1 / 2 \pi \Delta \nu$ much longer than the phonon lifetime, $\tau_{A}$, is required to ensure a stable pre-activation of the acoustic wave through optical interference between the pump and signal waves. The polarized output of the laser diode is split into two distinct channels to allow both the pump and signal waves to be derived from the same optical source [20]. The signal is generated in the upper channel, where an integrated electro-optic intensity modulator, driven by a microwave signal

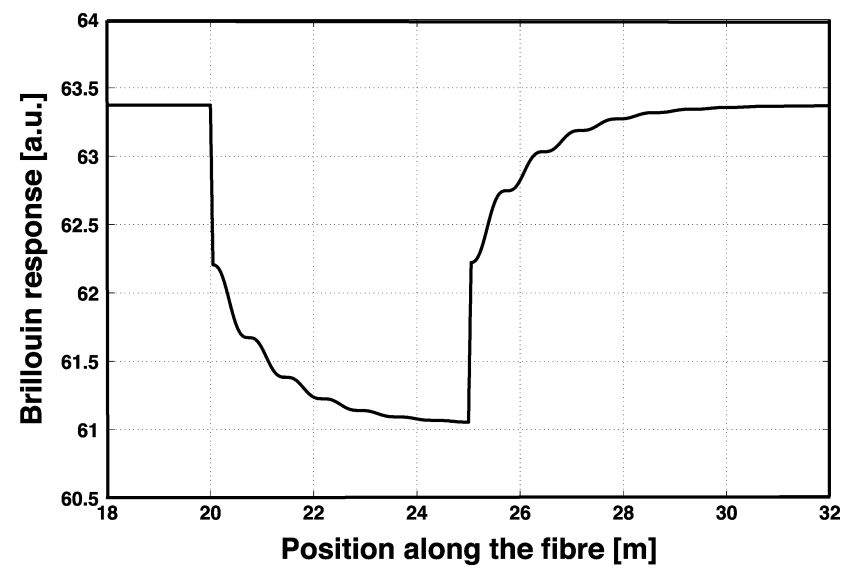

Fig. 4. The Brillouin signal from the $5 \mathrm{~m}$ section in a $20 \mathrm{~m}(10.73 \mathrm{GHz})-5 \mathrm{~m}$ $(10.86 \mathrm{GHz})-20 \mathrm{~m}(10.73 \mathrm{GHz})$ fiber, probed by a $500 \mathrm{ps} \pi$ phase shift pulse. Brillouin frequency is tuned at resonance for the $5 \mathrm{~m}$ section $(10.86 \mathrm{GHz})$. The fast fall time is accompanied by an exponential decrease to saturation. As the $\pi$ phase shift pulse leaves the $5 \mathrm{~m}$ section and enters of the $20 \mathrm{~m}$ one, a slow recovery of the signal follows a fast rise time. The ripples are due to the relative proximity of the Brillouin frequencies $(140 \mathrm{MHz})$ of the various sections, and reflect the non-zero contributions of the fairly long sections to the overall Brillouin signal.

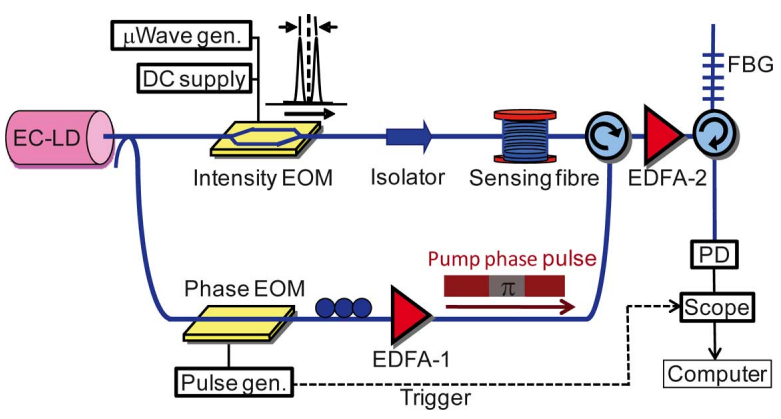

Fig. 5. Experimental setup of the optimized BEDS system: EC-LD: external cavity laser diode; EDFA: erbium-doped fiber amplifier; EOM: electro-optic modulator; FBG: fiber Bragg grating; PD: photodiode.

in a suppressed carrier configuration, creates sidebands, which are launched into one end of the sensing fiber. The lower channel is connected to the opposite end of the sensing fiber to launch a continuous pump wave that is periodically modulated by a phase modulator, to produce very short $\pi$ phase pulses. Electrical pulses from a fast pulse generator (500 ps minimum pulse duration, corresponding to a minimum spatial resolution of $5 \mathrm{~cm}$ ), are then applied to the phase modulator. The output light is controlled by a polarization controller to best align pump and signal polarizations to maximize the SBS interaction, and then boosted to $20 \mathrm{dBm}$ by a high power EDFA-1. In order to be not limited by the electronic noise of the wideband photodetector module, the signal at the output of the circulator, modified by the Brillouin interaction, is further amplified by EDFA-2. Finally, a fiber Bragg grating filter (bandwidth $10 \mathrm{GHz}$ ) is placed in front of the detector to eliminate the upper sideband from the signal and any residual and Rayleigh backscattered light from the pump.

In this setup, all stray forward propagating waves other than the signal, such as residual light due to the finite extinction ratio of the modulator, as well as spurious reflections and Rayleigh backscattering of the counter-propagating strong pump, all 
spectrally positioned at the pump frequency, are highly suppressed by the Bragg grating, thereby minimizing optical noise [19].

The Brillouin gain spectrum is determined by scanning the microwave generator around the Brillouin shift $\nu_{B}$. The net Brillouin gain is then measured by converting the optical signal using a DC-1 GHz amplified photodiode module (PD), followed by a high-pass electronic filter. The $1 \mathrm{GHz}$ bandwidth corresponds to a $350 \mathrm{ps}$ rise time that is sufficient to resolve a 500 ps transition. For each preset microwave frequency the signal is acquired in the time domain by a $4 \mathrm{GHz}$ digital oscilloscope, synchronously triggered by the $\pi$ shift pulse.

EDFA-2 was placed at the output of the circulator, rather than before the sensing fiber in the signal channel, in order to avoid pump depletion so that the intensity of the signal does not become comparable to that of the pump, thereby limiting the detection range and the sensing accuracy [21], [22]. On the other hand, the configuration of Fig. 5 will suffer more from the optical ASE noise generated by the amplifier, see Section V.

\section{EXPERIMENTAL RESULTS}

To study the performance of the BEDS system a test fiber comprising a $5 \mathrm{~cm}$ section of G652A fiber, spliced in the middle of a $40 \mathrm{~m}$ G652D fiber was fabricated. This creates an abrupt Brillouin frequency shift at the transition to the centimetric section from $10.730 \mathrm{GHz}$ (G652D fiber) to $10.860 \mathrm{GHz}$ (G652A fiber), as a simple consequence of the difference in core doping concentration, see Fig. 6(b) below. An averaging of 256 waveforms per frequency step was performed, resulting in a total acquisition time of about $8 \mathrm{~min}$. Most of this time was associated with the slow setting time of the microwave generator and the data transfer to the computer. The actual amount of time strictly needed to fully analyze the fiber (considering 256 averages and 256 frequency steps) is less than $10 \mathrm{~s}$.

Fig. 6(a) shows the enlarged top view of the 3-D distribution of the Brillouin response in the vicinity of the $5 \mathrm{~cm}$ fiber section and also the calculated Brillouin frequency shift, as a function of position (solid line) around the $5 \mathrm{~cm}$ fiber section. The central frequency of the Brillouin gain was determined by fitting the raw data to a Lorentzian distribution. The uncertainty was about $0.5 \mathrm{MHz}$ corresponding to a temperature accuracy of about $\pm 0.5^{\circ} \mathrm{C}$ and strain accuracy of about $\pm 8.75 \mu \varepsilon$. The figure clearly shows that each fiber section is fully resolved both spatially and spectrally. Measurements were performed using a 500 ps phase pulse $(5 \mathrm{~cm}$ resolution). The observed dynamic margin is quite large, indicating that a still higher spatial resolution is possible, albeit with a lower amplitude. The SNR is much improved when compared to our previous results obtained with a $10 \mathrm{~cm}$ spatial resolution [14]. This comes essentially from the fact that a standard DFB laser was used for those measurements, leading to a less efficient build-up of the acoustic wave and, consequently, a more noisy response.

We could experimentally confirm that the Brillouin gain spectrum remains undistorted for any pulse width, and that the linewidth of the measured spectrum obtained by BEDS is essentially independent of the pulse duration, as illustrated in Fig. 7(a). Clearly, this is not the case for a classical BOTDA method, where the linewidth is very dependent of the pulse

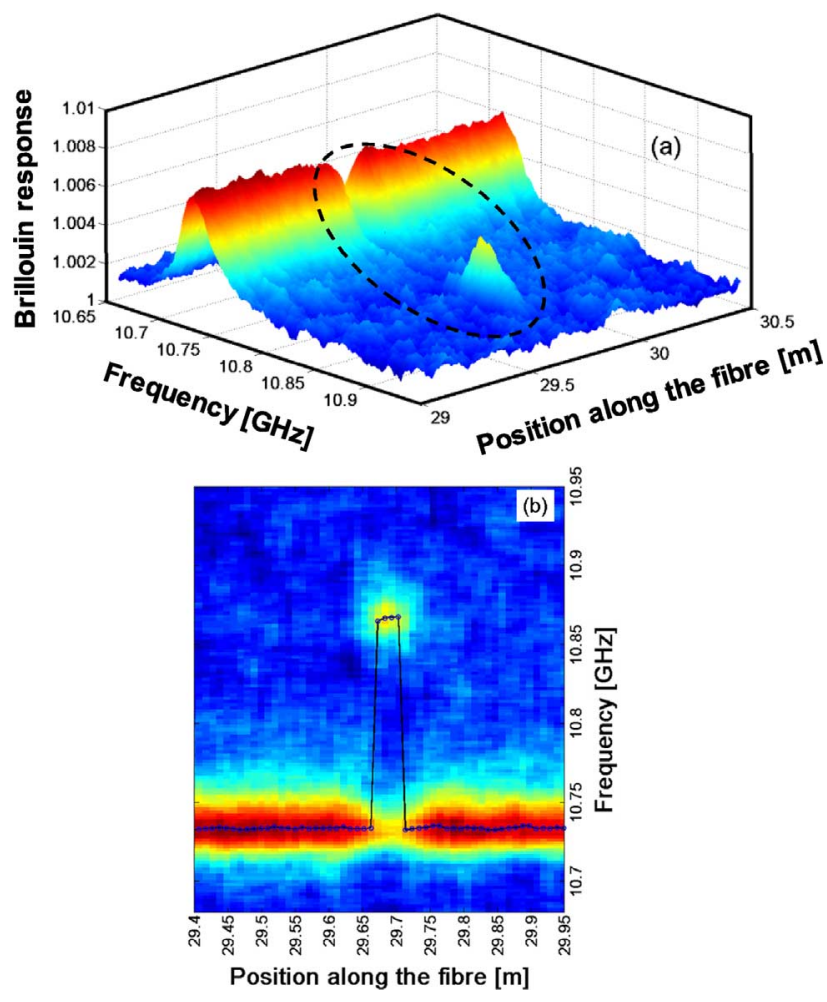

Fig. 6. (a) 3-D distribution of the Brillouin response along the sensing fiber as a function of frequency and position, (b) Enlarged top view of the 3-D distribution of the Brillouin gain in the vicinity of the $5 \mathrm{~cm}$ fiber section. Since the echo response manifests as an apparent loss the signal polarity has been inverted for clarity.

width, and increases as the pulsewidth decreases, as shown in Fig. 7(b).

\section{IsSues Related to the Continuous Activation of the ACOUSTIC FIELD}

While the underlying principle of the BEDS technique has been experimentally validated, its application to practical sensing requires some modifications. Actually the implementation of Fig. 5 suffers from the following penalties.

1) Continuous activation-So far, pre-activation of the acoustic field has been realized through the interference of a continuous signal with a continuous pump. However, this continuous interaction along the full length of the fiber gives rise to several detrimental effects:

a) Pump depletion, which limits both the sensing range and sensing accuracy [21], [22]. Actually, to avoid pump depletion in the setup of Fig. 5, EDFA-2 could not precede the fiber under test. Its current position, after the circulator comes at the expense of a less attenuated amplified spontaneous emission at the detector, resulting in higher signal-beat noise. More averaging could reduce the impact of this noise but requires longer acquisition times;

b) Spontaneous Brillouin noise-A continuous pump, energizing the whole length of the fiber generates at each section of the fiber a spontaneous Brillouin signal, centered around the local Brillouin frequency of the section. This accumulated parasitic signal 

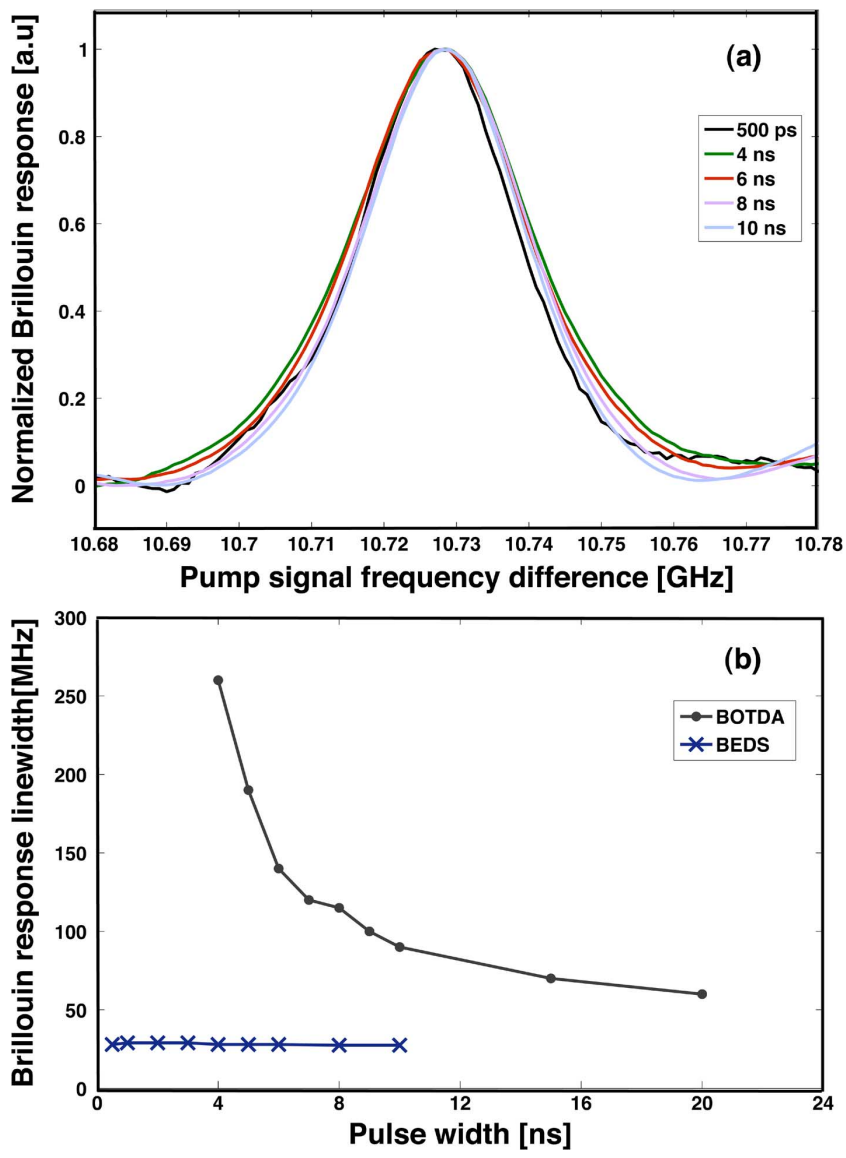

Fig. 7. (a) Spectral distribution of the Brillouin response measured locally by the Brillouin echo of a $\pi$-phase pulse, for a broad range of pulsewidth shorter than the acoustic lifetime, showing that the measured linewidth is unchanged and equal to the natural linewidth $\Delta \nu_{B}=27 \mathrm{MHz}$; (b) Measured Brillouin response linewidth as function of the pulsewidth for a classical BOTDA (Brillouin optical time domain analysis) and for the BEDS technique, showing the critical dependency of the linewidth on the pulsewidth in the case of the BOTDA.

interferes with the continuous probe wave, as it scans the frequency range of interest, to produce optical noise, see Fig. 8. For a long fiber with length-varying $\nu_{B}$, and a detector, which is spectrally wide open $(1 \mathrm{GHz})$ to accommodate fast $\beta$ pulses, this type of optical beat noise may limit the noise floor of the sensor [23], [24].

2) The second echo-As explained in Section II, the propagating $\beta$ pulse slightly modifies the acoustic field, resulting in a non-local response of the Brillouin sensor, i.e., the measured level of the $\beta$ pulse at a given spatial point is distorted by contributions from neighboring points. The longer the $\beta$ pulse, the more severe is the effect. Figs. 9-10 show experimental results for a $1 \mathrm{~ns} \pi$ phase pulse, probing a $1 \mathrm{~m}$ length fiber $\left(\nu_{B}=10.86 \mathrm{GHz}\right)$, placed in the middle of a $40 \mathrm{~m}$ fiber with $\nu_{B}=10.73 \mathrm{GHz}$. The 3-D distribution of the Brillouin response along the sensing fiber is shown in Fig. 9 as a function of both the frequency and spatial position. The spatial dependence of the Brillouin gain $\nu_{B}=10.86 \mathrm{GHz}$ is shown in Fig. 10(a) (i.e., a cut through Fig. 9 with the original polarity conserved) and favorably compares with Fig. 4 (Note that in the latter, the fiber seg-

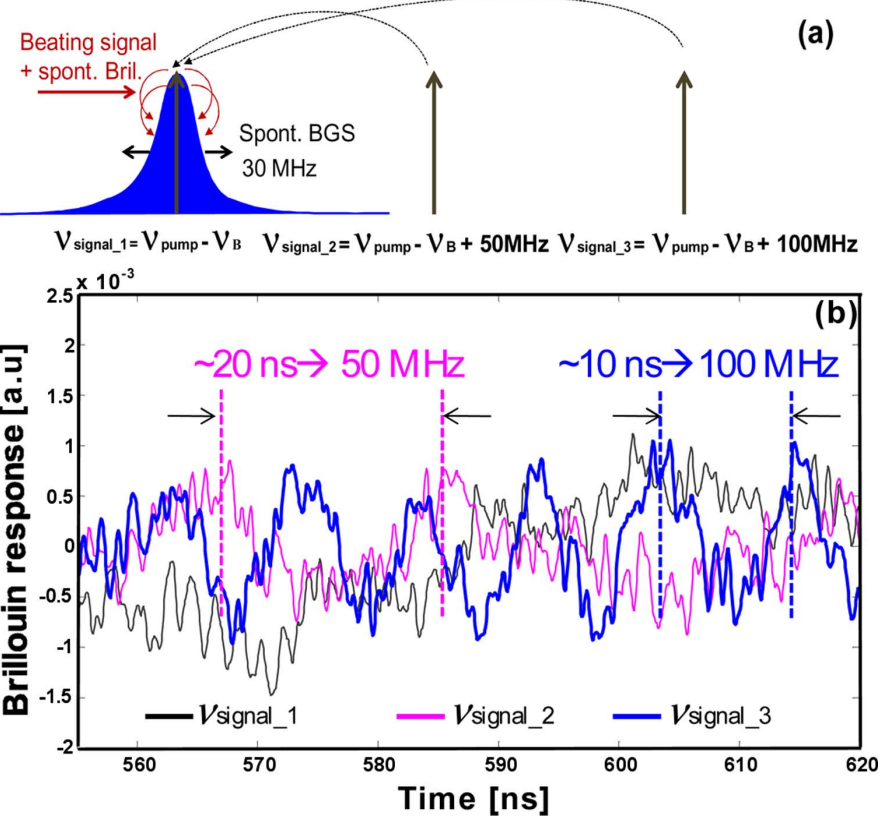

Fig. 8. (a) The scheme shows that the signal wave beats with the Brillouin spontaneous amplified emission from long homogeneous sections; The beat frequency is higher for signal frequencies spectrally more distant from the spontaneous amplified emission, giving rise to a noisy beat note jamming the response, as shown in (b).

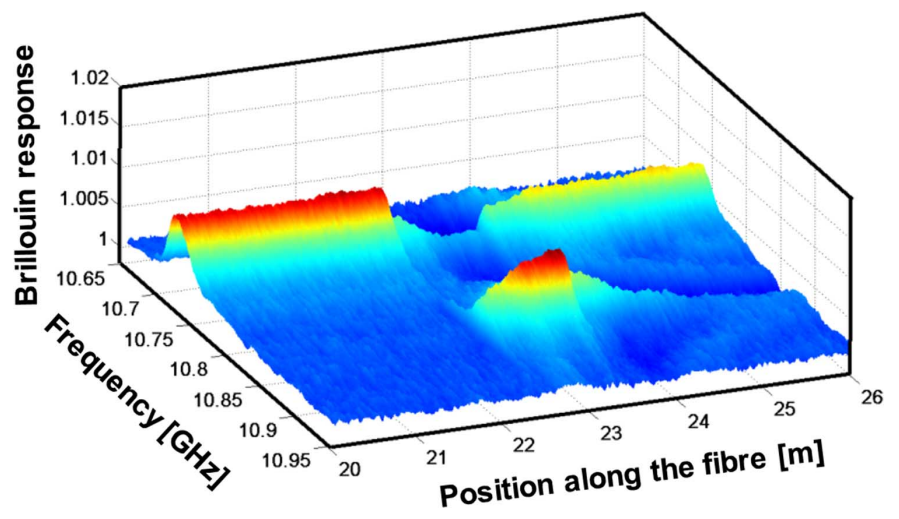

Fig. 9. 3D distribution of the Brillouin response along the sensing fiber as a function of the frequency and the position using a $1 \mathrm{~ns}$ p phase pulse. The fiber comprises a $1 \mathrm{~m}$ section with $\nu_{B}$ of $10.86 \mathrm{GHz}$, in the middle of $40 \mathrm{~m}$ of fiber with $\nu_{B}=10.73 \mathrm{GHz}$.

ment was chosen longer $(5 \mathrm{~m})$ in order to exhibit the saturation characteristics of the signal).

Instead of assuming a constant lower value throughout the $1 \mathrm{~m}$ section (excluding the very short $(\sim 10 \mathrm{~cm})$ fall and rise times), the signal demonstrates a slow descent towards a saturation value, not actually reached for the $1 \mathrm{~m}$ section. Furthermore, following the exit of the $\beta$ pulse, the signal returns to its proper value only after a slow recovery. As explained in Section II, this behavior is a manifestation of the second echo: the slow descent in the $1 \mathrm{~m}$ section originates from a $\beta$-induced perturbation of the acoustic field along a short segment of the $20 \mathrm{~m}$ fiber just preceding the $1 \mathrm{~m}$ section. Similarly, the $\beta$-modified acoustic field in the $1 \mathrm{~m}$ section is responsible for the slow recovery of the signal in the $20 \mathrm{~m}$ section following the $1 \mathrm{~m}$ section. Actually the time scale of saturation and recovery is on the order of $2 \times \tau_{A}$. 

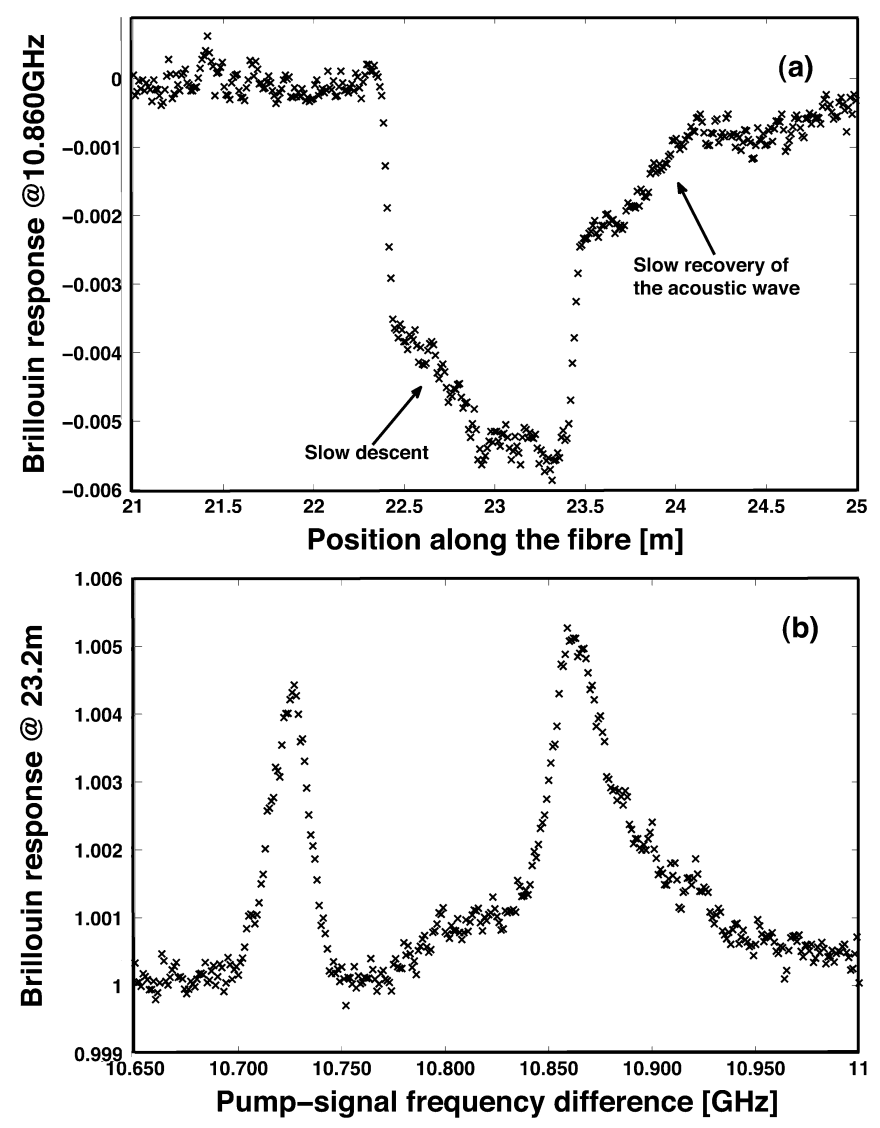

Fig. 10. Probing a $1 \mathrm{~m}$ section $\left(\nu_{B}=10.86 \mathrm{GHz}\right)$ in the middle of a $40 \mathrm{~m}$ fiber $\left(\nu_{B}=10.73 \mathrm{GHz}\right)$, using a $1 \mathrm{~ns} \pi$ phase pulse and a 256 time average for each temporal trace. (a) The Brillouin gain through the $1 \mathrm{~m}$ section at frequency $\nu_{B}=10.86 \mathrm{GHz},(1 \mathrm{~m}$ section at resonance). The marked slow descent of the signal at the beginning of the section, and the marked slow recovery of the signal as the $\pi$ phase shift pulse enters the subsequent $20 \mathrm{~m}$ section, both of the order of $\tau_{A} \sim 6 \mathrm{~ns}$, are manifestations of the second echo; (b) Measured Brillouin gain distribution within the $1 \mathrm{~m}$ section at position $23.2 \mathrm{~m}$ showing the high quality of the measured spectrum and the unwanted presence of the second echo. Since the $\pi$ phase shift pulse actually manifests as an apparent Brillouin loss, the signal polarity has been inverted for clarity.

For the same reason, the Brillouin response at $\nu_{B}=10.73 \mathrm{GHz}$, Fig. 9, still shows a non-zero signal in the $1 \mathrm{~m}$ section, resulting in an ambiguous spectrum measured well within the short segment, where the signal was supposed to show a single peak at $\nu_{B}=10.86 \mathrm{GHz}$, as illustrated in Fig. 10(b).

A differential pulsed pump approach, capable of alleviating the detrimental effects of the continuous pump, as well as eliminating the aftermath of the second echo, first successfully tested in [14] for BEDS and recently adapted for a bright pulse [16], is presented in the next section.

\section{The DifFerential Gain Method}

The effects of the second echo can be eliminated by extinguishing the pump at the end of the $\beta$ pulse (i.e., $\gamma=0$ in Fig. 1). Thus, in the absence of any pump light (no right term in (5a)), the back-propagating signal wave no longer experiences Brillouin interaction at all points where the $\beta$ pulse had already passed through. Using an additional intensity modulator in front of the phase modulator in the pump path of Fig. 5, the $\alpha$ pulse is

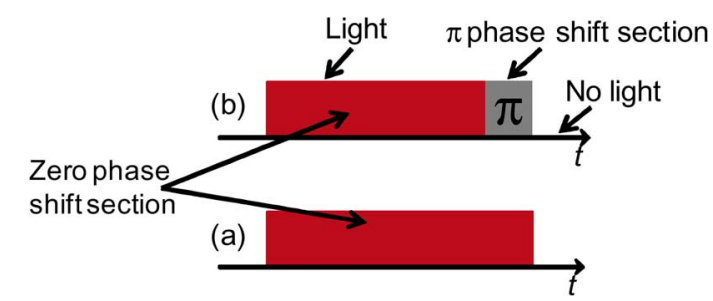

Fig. 11. Illustration of the pulse configuration, as used for the differential gain approach. (a) The probing pulse, comprising a short $\pi$ phase shift portion at the end of zero phase section; (b) A zero phase reference pulse.

also made finite, long enough ( $\sim 30 \mathrm{~ns})$ to substantially activate the acoustic wave but not too long in order to minimize pump depletion and optical beat noise (see Section V). Launching the pulse of Fig. 11(a), comprising a 30 ns long pulse, for which the last portion has been inverted ( $\pi$ phase shift) will actually activate the acoustic field and probe it without the parasitic second echo.

However, the unique contribution of the short $\pi$ phase shift portion of the pulse cannot be extracted unless extra measurements are taken.

In our differential gain method, first proposed in [14], two measurements are carried out: the first measurement is performed by using the pulse described in Fig. 11(b), while a second measurement records the response to a unipolar pulse, Fig. 11(a), of same length as the first pulse. Taking the difference between these two measurements results in a clean Brillouin trace, characterized by high spatial resolution, high spectral resolution, and long range capabilities.

Figs. 12-13 show the application of the technique to the 20 m-1 m-20 m fiber of Figs. 9-10. As described above, measurements were carried out in two steps. First, the pump power was turned on for $30 \mathrm{~ns}$, resulting in a classical Brillouin trace with a low spatial resolution of only $3 \mathrm{~m}$. Then the $30 \mathrm{~ns}$ compound pulse, comprising the original $29.5 \mathrm{~ns}$ zero phase and an appended $0.5 \mathrm{~ns} \pi$ phase shift pulse, was launched into the fiber and this new obtained trace was subtracted from the first one, giving the resulting response of the sole ultra short phase pulse. The rise/fall time of the pulses is particularly critical to secure the spatial resolution and was limited to 200 ps by our pulse generator, reasonably shorter than the $\pi$ phase shift pulse width. No second echo is observed in Figs. 12-13 and the Brillouin sensing is actually strictly local. Using the same pulse parameters, the method has been also applied to a $5 \mathrm{~km}$ fiber $\left(\nu_{B}=10.42 \mathrm{GHz}\right)$, where a $5 \mathrm{~cm}$ fiber with $\nu_{B}=10.30 \mathrm{GHz}$, was spliced near the far end of the long fiber. Since SBS is inherently dependent on the polarization, a passive polarization scrambler was placed after the pump to achieve non-vanishing average gain at every fiber location, regardless of the actual signal polarization at the same spatial point. The differential pulse-width pair technique described in [16] can be seen as a simplification of the differential method here detailed, in which the $\pi$ phase shift pulse is replaced by a dark pulse making the actual pump pulse shorter. In Fig. 14 a comparative measurement of the response of these two differential techniques is presented, showing that the phase pulse actually optimizes the response by improving the contrast by a factor 2, as expected from the analysis in Section II. 


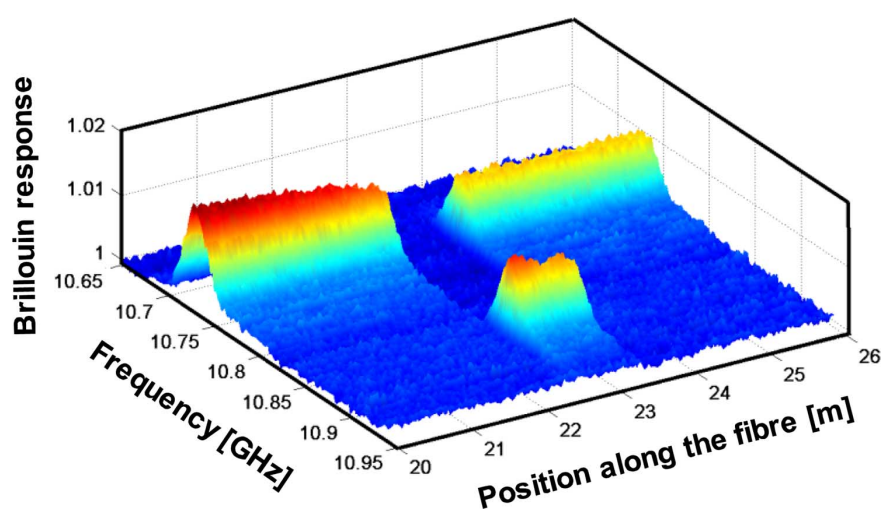

Fig. 12. A 3-D map of the Brillouin loss in the $20 \mathrm{~m}-1 \mathrm{~m}-20 \mathrm{~m}$ fiber of Fig. 9, as obtained from the differential gain technique. No second echoes are observed.
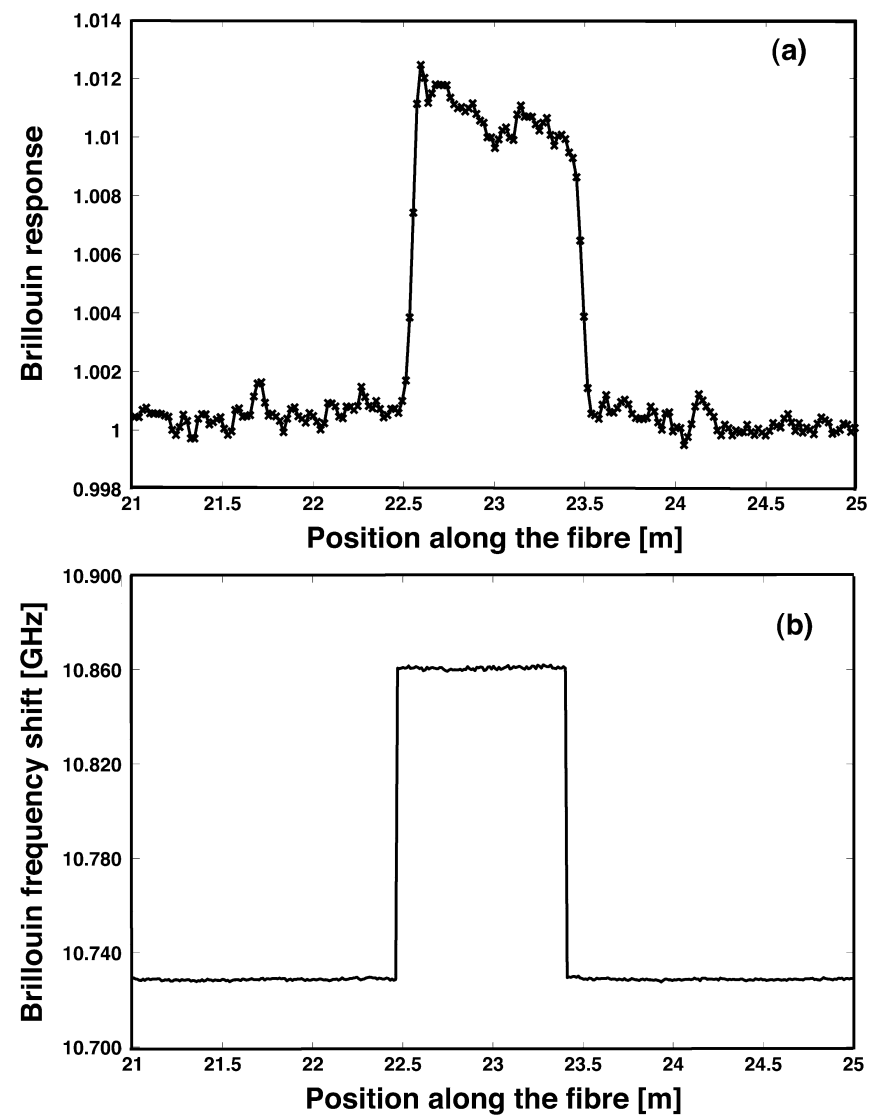

Fig. 13. Using the differential gain technique to probe a $1 \mathrm{~m}$ section $\left(\nu_{B}=\right.$ $10.86 \mathrm{GHz})$ in the middle of a $40 \mathrm{~m}$ fiber $\left(\nu_{B}=10.73 \mathrm{GHz}\right)$, using a $500 \mathrm{ps}$ p phase pulse and a 256 time averages of each temporal trace. (a) Compare the very clean Brillouin gain trace around the $1 \mathrm{~m}$ section at the $1 \mathrm{~m}$ section frequency $\left(\nu_{B}=10.86 \mathrm{GHz}\right)$ with that of Fig. 10(a). (b) Brillouin frequency shift as a function of position in the fiber.

Time traces shown in Fig. 15 document consecutive measurements performed along the $5 \mathrm{~km}$ long fiber with (B) and without (A) the $0.5 \mathrm{~ns} \pi$ phase pulse, together with their difference (bottom curve and inset). Fig. 16 depicts a close-up view of the (A-B) curve of Fig. 15 around the position of the $5 \mathrm{~cm}$ section, exhibiting excellent spatial and spectral resolution. Fig. 16(a) shows the gain as a function of the position around the $5 \mathrm{~cm}$ section, when the frequency is tuned to be at the peak frequency of the short segment, while Fig. 16(b) describes the Brillouin

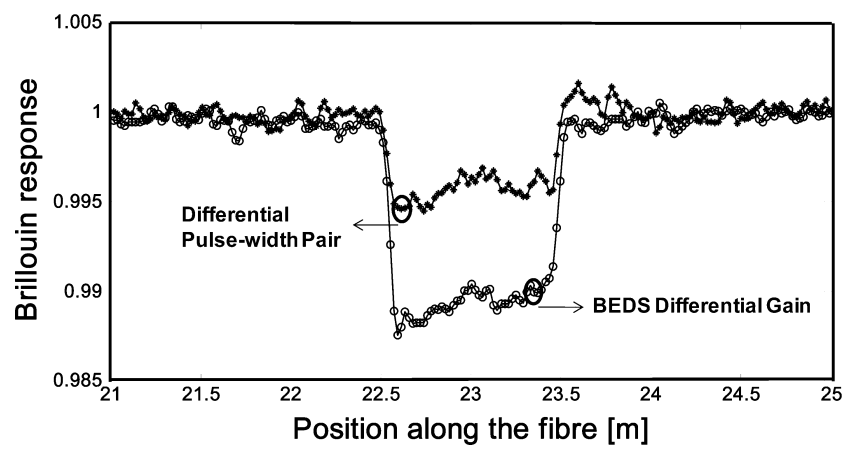

Fig. 14. Comparative Brillouin response of the BEDS differential gain approach and the differential pulse-width pair technique [16], obtained under identical power and spatial resolution conditions $(5 \mathrm{~cm})$, clearly showing the optimized BEDS response by a $3 \mathrm{~dB}$ contrast improvement.

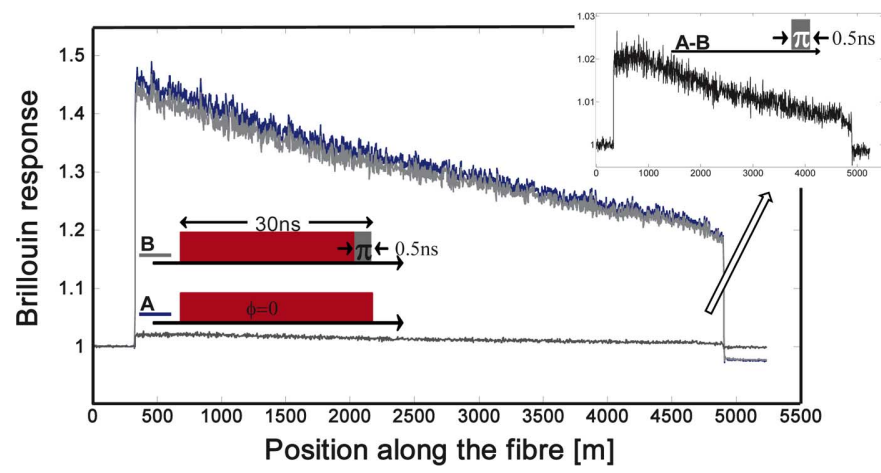

Fig. 15. Two measurements without (A) and with (B) $0.5 \mathrm{~ns} \pi$ phase pulse along the $5 \mathrm{~km}$ long fiber. Inset: the difference between the two measurements $\mathrm{A}$ and $\mathrm{B}$ showing the signature of the fiber equivalent to the response of the $0.5 \mathrm{~ns}$ phase pulse. Measurements were performed using 256 times averaging of each temporal trace.

frequency shift around the $5 \mathrm{~cm}$ fiber section, as a function of distance, after processing of the raw traces. The $5 \mathrm{~cm}$ section is very well resolved and the accuracy of the measurement is calculated to be $\pm 3 \mathrm{MHz}$ for 2 standard deviations $\sigma$, corresponding to $\pm 3^{\circ} \mathrm{C}$.

A 3-D view is provided in Fig. 17 where again, the $5 \mathrm{~cm} \mathrm{sec-}$ tion is spatially well resolved, while preserving the $\sim 30 \mathrm{MHz}$ natural linewidth of the Brillouin gain spectrum.

\section{CONCLUSION}

Brillouin echo distributed sensing (BEDS) has proved to be a powerful solution to realize sub-metric spatial resolutions in Brillouin distributed measurements. An optimized configuration, using $\pi$-phase pump pulses, was experimentally tested down to a spatial resolution of $5 \mathrm{~cm}$, with a clear margin for further improvement down to a real centimetric spatial resolution. This optimized configuration will produce the best contrast independently of the pulse intensity, with a factor 2 of improvement compared to other techniques based on the same approach. This extends the dynamic range by $3 \mathrm{~dB}$, which corresponds in standard loss conditions to a $5 \mathrm{~km}$ extension of the sensing range.

Major difficulties related to the restoring of the acoustic wave that manifests as a secondary trailing echo in the response have been successful alleviated using a differential gain approach. 

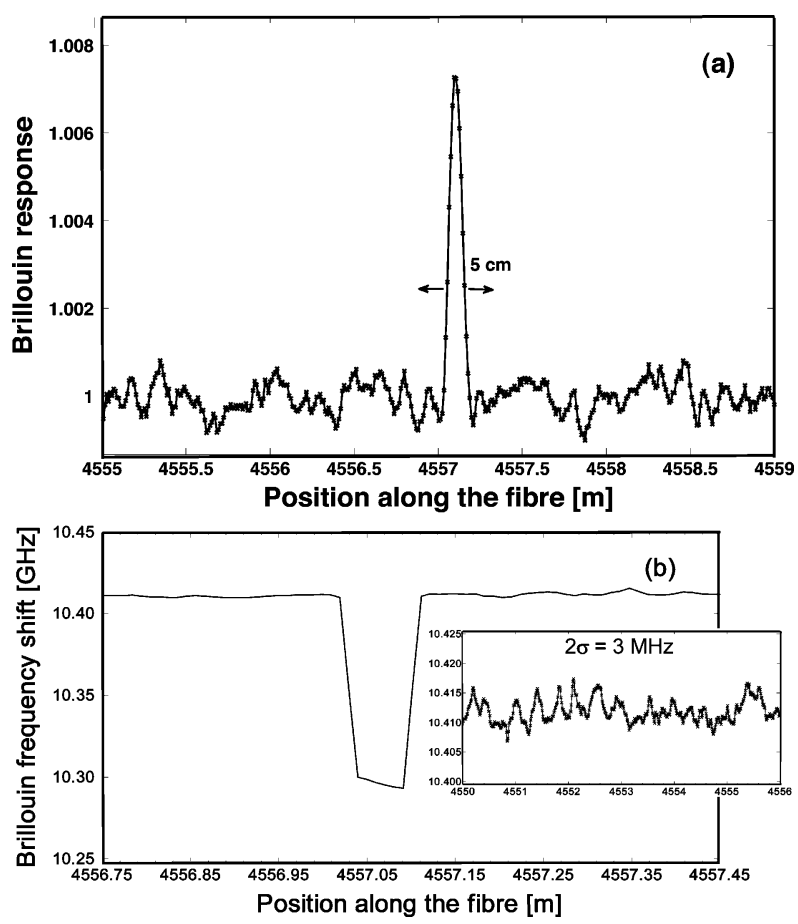

Fig. 16. (a) Brillouin response as a function of distance in the vicinity of the $5 \mathrm{~cm}$ fiber section; (b)Brillouin frequency shift as a function of distance at the same positions.
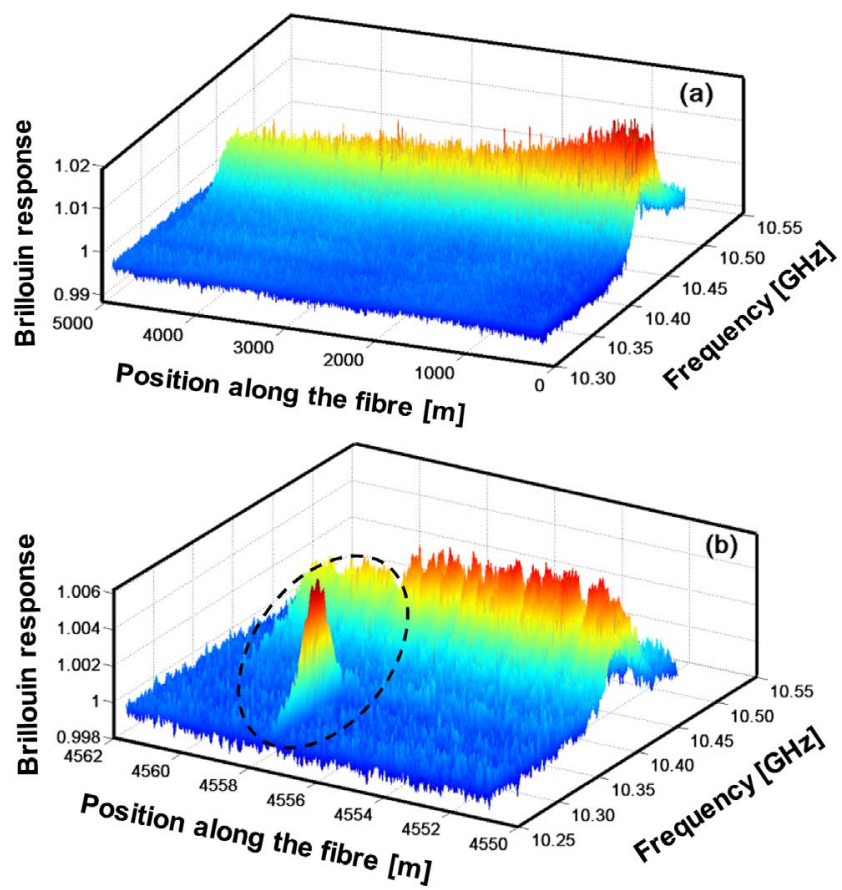

Fig. 17. (a) 3-D spectral distribution of the response as function of the position all over a $5 \mathrm{~km}$ fiber realized with a $5 \mathrm{~cm}$ spatial resolution. The $5 \mathrm{~cm}$ segment is too small to be distinguished; (b) Magnified view of the 3-D distribution of the Brillouin response around the $5 \mathrm{~cm}$ fiber section, showing that this segment at end of the $5 \mathrm{~km}$ fiber is fully resolved both in space and frequency.

This has limited excessive pump depletion and made it possible to extend the range to $5 \mathrm{~km}$, which was limited to much less than $1 \mathrm{~km}$ using a continuous pump. This corresponds to 100 '000 independent resolved points along the fiber and a range extension to $10 \mathrm{~km}$ looks perfectly feasible, so that a system with one million resolved points along a standard single mode fiber is likely to be proposed in a near future. It appears that BEDS systems can become a key and powerful configuration for the next generation of Brillouin fiber distributed sensors, combining simplicity in the hardware implementation, kilometric sensing ranges and centimetric spatial resolution, with a temperature/strain resolution unchanged when compared to a classical BOTDA system.

\section{REFERENCES}

[1] T. Kurashima, T. Horuguchi, and M. Tateda, "Distributed-temperature sensing using stimulated Brillouin scattering in optical silica fibers," Opt. Lett., vol. 15, pp. 1038-1040, 1990.

[2] X. Bao, D. J. Webb, and D. A. Jackson, "32-Km distributed temperature sensor using Brillouin loss in optical fiber," Opt. Lett., vol. 18, pp. 1561-1563, 1993.

[3] M. Nikles, L. Thévenaz, and P. A. Robert, "Simple distributed fiber sensor based on Brillouin gain spectrum analysis," Opt Lett., vol. 21, no. 10, May 1996.

[4] T. Kurashima, T. Horiguchi, H. Izumita, S. Furukawa, and Y. Koyamada, "Brillouin optical fiber time domain reflectometry," IEICE Trans. Commun., vol. E76-B, no. 4, pp. 382-390, 2008.

[5] A. Fellay, L. Thévenaz, M. Facchini, M. Nikles, and P. Robert, "Distributed sensing using stimulated Brillouin scattering: Towards ultimate resolution," in Proc. 12th Int. Conf. Opt. Fiber Sensors, 1997, pp. 324-327.

[6] G. Agrawal, Nonlinear Optics, 3rd ed. New York: Academic, 2008, ch. 9 .

[7] D. Garus, T. Gogolla, K. Krebber, and F. Schliep, "Brillouin opticalfiber frequency-domain analysis for distributed temperature and strain measurements," J. Lightw. Technol., vol. 15, no. 4, pp. 654-662, Apr. 1997.

[8] K. Hotate and M. Tanaka, "Distributed fiber Brillouin strain sensing with 1-cm spatial resolution by correlation-based continuous-wave technique," IEEE Photon. Technol. Lett., vol. 14, no. 2, pp. 179-181, Feb. 2002.

[9] X. Bao, A. Brown, M. DeMerchant, and J. Smith, "Characterization of the Brillouin-loss spectrum of single-mode fibers by use of very short (10-ns) pulses," Opt. Lett., vol. 24, pp. 510-512, 1999.

[10] V. Lecoeuche, D. J. Webb, C. N. Pannell, and D. A. Jackson, "Transient response in high-resolution Brillouin-based distributed sensing using probe pulses shorter than the acoustic relaxation time," Opt. Lett., vol. 25, pp. 156-158, 2000.

[11] L. Zou, X. Bao, Y. Wan, and L. Chen, "Coherent probe-pump-based Brillouin sensor for centimeter-crack detection," Opt. Lett., vol. 30, pp. 370-372, 2005.

[12] A. W. Brown and B. G. Colpitts, "Dark-Pulse Brillouin optical timedomain sensor with 20-mm spatial resolution," J. Lightw. Technol., vol. 25, no. 2, pp. 381-386, Feb. 2007.

[13] K. Kishida and C.-H. Li, , J. P. Or, H. Li, and Z. D. Duan, Eds., "Pulse pre-pump-BOTDA technology for new generation of distributed strain measuring system," in Structural Health Monitoring and Intelligent Infrastructure. London, U.K.: Taylor \& Francis, 2006, vol. 1, pp. 471-477.

[14] L. Thévenaz and S. M. Foaleng, "Distributed fiber sensing using Brillouin echoes," in Proc. 19th Int. Conf. Opt. Fiber Sensors, Perth, WA, Australia, 2008, pp. 70043N-70044, SPIE.

[15] S. M. Foaleng, J.-C. Beugnot, and L. Thévenaz, "Optimized configuration for high resolution distributed sensing using Brillouin echoes," in Proc. 20th Int. Conf. Opt. Fiber Sens., Edinburgh, U.K., 2009, vol. 7503, pp. 75032C-4, SPIE.

[16] W. Li, X. Bao, Y. Li, and L. Chen, "Differential pulse-width pair BOTDA for high spatial resolution sensing," Opt. Exp., vol. 16, pp. 21616-21625, 2008.

[17] R. W. Boyd, Nonlinear Optics, 4th ed. New York: Academic, 2008, ch. 9 .

[18] L. Thévenaz and J.-C. Beugnot, "General analytical model for distributed Brillouin sensors with sub-meter spatial resolution," in Pro. 20th Int. Conf. Optical Fiber Sens. SPIE, Edinburgh, UK, 2009, p. 7503

[19] S. Diaz, S. M. Foaleng, M. Lopez-Amo, and L. Thévenaz, "A highperformance optical time-domain Brillouin distributed fiber sensor," IEEE Sensors J., vol. 8, no. 1, pp. 1268-1272, Jan. 2008. 
[20] M. Niklès, L. Thévenaz, and P. Robert, "Brillouin gain spectrum characterization in single-mode optical fibers," J. Lightw. Technol., vol. 15, no. 10, pp. 1842-1851, Oct. 1997.

[21] T. Horiguchi, K. Shimizu, T. Kurashima, M. Tateda, and Y. Koyamada, "Development of a distributed sensing technique using Brillouin scattering," J. Lightw. Technol., vol. 13, no. 7, pp. 1296-1302, Jul. 1995.

[22] E. Geinitz, S. Jetschke, U. Röpke, S. Schröter, R. Willsch, and H. Bartelt, "The influence of pulse amplification on distributed fiber-optic Brillouin sensing and a method to compensate for systematic errors," Meas. Sci. Technol., vol. 10, pp. 112-116, 1999.

[23] R. W. Tkach and A. R. Chraplyvy, "Fiber Brillouin amplifiers," Opt. Quantum Electron., vol. 21, no. 1, pp. 105-112, Jan. 1989.

[24] N. A. Olsson and J. P. Van Der Ziel, "Characteristics of a semiconductor laser pumped Brillouin amplifier with electronically controlled bandwidth," J. Lightw. Technol., vol. 1, pp. 147-150, 1987.

Stella Mafang Foaleng received the degree of Dottoressa in Ingegneria de l'informazione from the Università degli studi di Perugia (UNIPG), Italy, in 2003, the M.Sc. degree in electrical and electronic engineering from The Swiss Federal Institute of Technology of Lausanne (EPFL), Switzerland, in 2006, where she is currently working towards the Ph.D. degree.

Her research topics include nonlinear fiber optics and Brillouin scattering distributed fiber optic sensors.

Moshe Tur (M'87-SM-94-F'98) received the B.Sc. degree in mathematics and physics, from the Hebrew University, Jerusalem, Israel, in 1969, the M.Sc. degree in applied physics from the Weizmann Institute of Science, Rehovot, Israel, in 1973, and the Ph.D. degree from Tel-Aviv University, Tel-Aviv, Israel, in 1981.

He is presently the Gordon Professor of Electrical Engineering at the School of Electrical Engineering of Tel-Aviv University, Tel-Aviv, Israel, where he has established a fiber-optic sensing and communication laboratory. He authored or co-authored more than 300 journal and conference technical papers with emphasis on fiber-optic sensing (with current emphasis on Structural health Monitoring, using fiber Bragg gratings and the Brillouin effect), polarization mode dispersion, microwave photonics, and advanced fiber-optic communication systems.

Dr. Tur is a Fellow of the Optical Society of America.
Jean-Charles Beugnot received the M.Sc. degree and the Ph.D. degree in electronics studies from the university of Franche-Comté, France, in 2004 and 2007 , respectively.

Since 2008, he is working as a post doctoral researcher in the group for fiber optics in the Swiss Federal Institute of Technology of Lausanne (EPFL), Lausanne, Switzerland. His research interests focus on fiber optic metrology and nonlinear effects in photonics crystal fibers.

Luc Thévenaz received the M.Sc. degree in 1982 and the Ph.D. degree in physics in 1988, both from the University of Geneva, Switzerland.

In 1988 he joined the Swiss Federal Institute of Technology of Lausanne (EPFL), Lausanne, Switzerland, where he currently leads a research group involved in photonics, namely fiber optics and optical sensing. Research topics include Brillouin-scattering fiber sensors, slow and fast light, nonlinear fiber optics and laser spectroscopy in gases. He achieved with his collaborators the first experimental demonstration of optically-controlled slow \& fast light in optical fibers, and is at the origin of innovative configurations for Brillouin distributed fiber sensors, such as generation of the signal wave using modulation sidebands and Brillouin dynamic gratings. During his career he stayed at Stanford University, at the Korea Advanced Institute of Science and Technology (KAIST), at Tel Aviv University and at the University of Sydney. In 2000 he co-founded the company Omnisens that is developing and commercializing advanced photonic instrumentation. He is the author or coauthor of some 280 publications and 5 patents.

Prof. Thévenaz is Chairman of the European COST Action 299 "FIDES: Optical Fibers for New Challenges Facing the Information Society." 